\title{
Spine Radiosurgery in the Management of Renal Cell Carcinoma Metastases
}

\author{
Neil K. Taunk, MD, MSa; Daniel E. Spratt, MDª Mark Bilsky, MDª ; and Yoshiya Yamada, MDa
}

\begin{abstract}
Renal cell carcinoma (RCC) presents an interesting challenge in radiation oncology. Improved systemic therapy has significantly prolonged survival. Modern imaging has allowed practitioners to effectively identify patients with oligometastatic disease. Conventionally fractionated radiation therapy is a first-line treatment option for palliation of bone metastases, including the spine, but has limited efficacy and durability. Conventional treatment may not be sufficient in metastatic RCC because of the disease's relative radioresistance. Improved technology, including custom immobilization and on-board treatment imaging, has allowed ultra-highdose radiation therapy, or stereotactic radiosurgery (SRS), to effectively treat metastatic disease in the spine. Safety and efficacy have already been established for intracranial disease and data are emerging for extracranial metastasis. Spine SRS offers local control rates and durable pain improvement in up to $90 \%$ of patients. Many series have already reported its effectiveness, and prospective multi-institutional trials are underway. Spine SRS should be strongly considered in select patients with refractory or oligometastatic disease. (J Natl Comp Canc Netw 2015;13:801-809)
\end{abstract}

\section{NCCN: Continuing Education}

\section{Accreditation Statement}

This activity has been designated to meet the educational needs of physicians and nurses involved in the management of patients with cancer. There is no fee for this article. No commercial support was received for this article. The National Comprehensive Cancer Network

From the Departments of a Radiation Oncology and ${ }^{b}$ Neurosurgery, Memorial Sloan Kettering Cancer Center, New York, New York. Submitted January 23, 2015; accepted for publication May 7, 2015. Dr. Bilsky had disclosed that he is on the advisory board for Varian Medical Systems, Inc., is on the cervical development team and a course instructor for Globus Medical Inc., and receives royalties from DePuy Synthes. Dr. Yamada has disclosed that he is a consultant for Varian Medical Systems, Inc. The remaining authors have disclosed that they have no financial interests, arrangements, affiliations, or commercial interests with the manufacturers of any products discussed in this article or their competitors.

Correspondence: Yoshiya Yamada, MD, Department of Radiation Oncology, Memorial Sloan Kettering Cancer Center, 1275 York Avenue, New York, NY 10065. E-mail: yamadaj@mskcc.org
(NCCN) is accredited by the ACCME to provide continuing medical education for physicians.

NCCN designates this journal-based CME activity for a maximum of 1.0 AMA PRA Category 1 Credit(s) TM. Physicians should claim only the credit commensurate with the extent of their participation in the activity.

NCCN is accredited as a provider of continuing nursing education by the American Nurses Credentialing Center's Commission on Accreditation.

This activity is accredited for 1.0 contact hour. Accreditation as a provider refers to recognition of educational activities only; accredited status does not imply endorsement by NCCN or ANCC of any commercial products discussed/displayed in conjunction with the educational activity. Kristina M. Gregory, RN, MSN, OCN, is our nurse planner for this educational activity.

All clinicians completing this activity will be issued a certificate of participation. To participate in this journal CE activity: 1) review the learning objectives and author disclosures; 2 ) study the education content; 3) take the posttest with a $66 \%$ minimum passing score and complete the evaluation at http://education.nccn.org/ node/68461; and 4) view/print certificate.

Release date: June 17, 2015; Expiration date: June 17, 2016

\section{Learning Objectives}

Upon completion of this activity, participants will be able to:

- Discuss the rationale and current data to support SRS in patients with metastatic RCC

- Identify those patients with metastatic RCC who may benefit the most from SRS treatment

EDITOR

Kerrin M. Green, MA, Assistant Managing Editor, JNCCN_Journal of the National Comprehensive Cancer Network

Ms. Green has disclosed that she has no relevant financial relationships.

\section{CE AUTHORS}

Deborah J. Moonan, RN, BSN, Director, Continuing Education, has disclosed that she has no relevant financial relationships.

Ann Gianola, MA, Manager, Continuing Education Accreditation \& Program Operations, has disclosed that she has no relevant financial relationships.

Kristina M. Gregory, RN, MSN, OCN, Vice President, Clinical Information Operations, has disclosed that she has no relevant financial relationships. Rashmi Kumar, PhD, Senior Manager, Clinical Content, has disclosed that she has no relevant financial relationships. 


\section{Renal Cell Carcinoma and the Case for Local Therapy for Metastatic Disease}

Renal cell carcinoma (RCC) is the seventh most common malignancy in the United States. An estimated 63,000 new RCC diagnoses were made in 2014, resulting in approximately 14,000 deaths. Fortunately, the survival rate of these patients has increased substantially, with an increased 5-year overall survival of $73 \%$ in the period from 2003 to $2009 .{ }^{1}$ At presentation, $25 \%$ of patients will demonstrate metastases, and $25 \%$ who undergo nephrectomy for localized disease will ultimately develop recurrent or metastatic tumor. ${ }^{1}$

Targeted therapies directed against vascular endothelial growth factor (VEGF) have revolutionized the management of metastatic RCC. Drugs include bevacizumab and tyrosine kinase inhibitors, such as sunitinib, sorafenib, pazopanib, and axitinib. Before targeted therapies were introduced, the incidence of skeletal events, such as spinal cord compression and pathologic fractures, approached $85 \%$ and portended a poor prognosis. ${ }^{2}$ Inclusion of these events improved a predictive model developed at Memorial Sloan Kettering Cancer Center (MSKCC) to characterize prognosis in patients with RCC. ${ }^{3,4}$ Even with these advances in systemic treatment, radiation therapy (RT) continues to play a significant role in managing patients with metastatic disease to the brain and spine. RT is used for palliation of painful bone metastases, spinal cord compression, and brain metastases. Significant technologic advances in RT, including intensity-modulation RT, image-guidance RT, stereotactic body RT (SBRT), and stereotactic radiosurgery (SRS), have allowed radiation oncologists to deliver high doses with accuracy within millimeters. Durable local control may exceed 90\% with SRS, independent of histology, providing selected patients with excellent palliation and long-term disease-free intervals. ${ }^{5}$ This article reviews the rationale, data, and indications for SRS of the spine, for which local control is critical in palliation and prevention of neurologic injury. In addition, commonly reported toxicities and the role of SRS are discussed in light of improving systemic therapy.

\section{Role of RT in RCC}

RT has little role in treating primary localized RCC. Surgery is the primary modality for localized RCC and select metastatic cases for cytoreduction. ${ }^{6}$ Two prospective trials examining preoperative RT showed no significant survival benefit, with one even showing inferior survival with RT.7,8 Early prospective studies failed to show local control or a survival benefit for postoperative RT. ${ }^{9}$ A later postoperative RT meta-analysis showed improved local control with no overall or disease-free survival benefit. ${ }^{10}$ Given these studies, both preoperative and postoperative RT are not indicated in the management of localized RCC.

Although conventionally fractionated RT has shown little clinical utility in the neoadjuvant or adjuvant setting, SBRT, discussed further later, has been used in primary recurrent disease or in medically inoperable patients. Wersäll et $\mathrm{al}^{11}$ reported on a subgroup of 8 patients with inoperable primary RCC treated with SBRT. These patients had a median survival of more than 58 months, and 7 of 8 had no recurrence at last follow-up. In a series of 9 patients who refused surgery for localized or primary recurrent RCC (2 with bilateral disease), SBRT resulted in no in-field failures. ${ }^{12}$ Considering high doseper-fraction RT appears locally effective in these clinical scenarios, further study is warranted. ${ }^{13}$

Radiation is the principle local treatment for intracranial and extracranial metastases. Conventionally fractionated RT may be ablative with definitive doses, but normal tissue tolerance limits the use of high-dose RT. A typical palliative schedule in conventional radiation consists of 8 Gy x 1, 4 Gy x 5, or 3 Gy x 10 fractions, which is suboptimal for some tumor histologies, such as RCC. ${ }^{14}$ SRS provides ablative doses of highly conformal radiation to tumor targets in 1 to 5 treatments (Figure 1). In the era of targeted therapy and improved survival, SRS offers durable local control for patients with metastatic RCC (Figure 2).

\section{Relative Radioresistance of RCC and the Case for Dose Escalation}

Data suggest that RCC is a relatively radioresistant disease. In the linear quadratic radiobiologic model of cell survival, the $\alpha / \beta$ ratio describes the curvature of the cell survival curve. Cells thought to have an $\alpha / \beta$ ratio higher than 10 , including tumor and rapidly proliferating normal tissue, are considered relatively radiosensitive and more susceptible to low-dose radiation. Late-responding tissues, including normal tissue, are thought to have an $\alpha / \beta$ ratio lower than 3. Cells with lower $\alpha / \beta$ ratios may require high dose-per-fraction radiation doses for response. Notably, some cancers, including prostate cancer, may have instead have a low $\alpha / \beta$ ratio. ${ }^{15}$ 
Spine Radiosurgery in Renal Cell Carcinoma

In A498 and Caki-1 human RCC cell lines in nude mouse xenografts and in vitro studies, the effect of RT was modest at approximately 2 Gy per fraction, but pronounced at more than 6 Gy. The $\alpha / \beta$ ratio was 6.9 and 2.6 for the Caki-1 and A498 cell lines, respectively, indicating that these lines are relatively radioresistant. ${ }^{16}$ Deschavanne et $\mathrm{al}^{17}$ found that RCC was the most radioresistant cell line of multiple histologies. Nude mice implanted with the same A498 RCC cell line irradiated with 48 Gy in 3 fractions showed that the disease responded well to hypofractionated RT, with extensive tumor necrosis. ${ }^{18}$ This study did not compare conventionally fractionated versus high-dose RT, but showed that high-dose RT is tumoricidal in RCC.

Conventionally fractionated RT mediates DNA damage in cancer cells leading to mitotic catastrophe. Single-fraction RT and high-dose hypofractionated RT may act on an entirely different mechanism, instead causing rapid endothelial cell apoptosis and microvascular damage. ${ }^{19}$ Immediately after high-dose RT, ceramide-mediated signaling of tumor necrosis factor- $\alpha$ and FasL-mediated apoptosis of cells occurs..$^{20}$ This continues to be an active area of study, but SRS is likely tumoricidal due to ablation of disease.

Dose escalation using high biologically effective dose (BED) radiation may overcome presumed radioresistance from conventionally fractionated treatment. Based on the linear quadratic model of cell response, the BED is commonly used to calculate isoeffective RT schedules. The model accounts for early effect (tumor tissue), late effect (normal tissue), and inherent differences in radiosensitivity. Studies using this model to determine BED indicated that higher equivalent doses may yield improved outcomes. An early study showed that higher BED (as a continuous variable) independently predicted overall response in palliative radiotherapy in RCC. ${ }^{21}$ This study assumed a $\alpha / \beta$ ratio of 10 for tumor, compared with the data reported previously that empirically showed a lower $\alpha / \beta .{ }^{16}$ Although the linear-quadratic model is reasonable to use with high-dose RT, potentially up to $18 \mathrm{~Gy}$ per fraction, further refinements in the model may be a better predictor of the effects of SRS..$^{22,23}$ The universal survival curve incorporates the linear-quadratic and the multitarget models to account for vascular and stromal damage caused by ablative RT. ${ }^{24}$

Compared with conventionally fractionated regimens, SBRT often results in higher BED in fewer

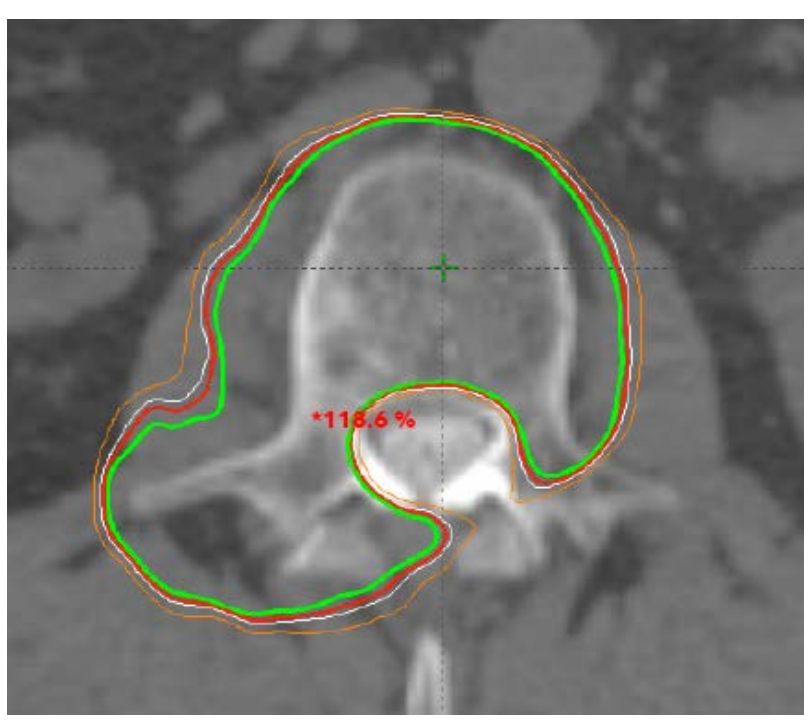

Figure 1 59-year-old man treated with single fraction (24 Gy) spine stereotactic radiosurgery for $\mathrm{L} 2$ body and pedicle disease, showing highly conformal doses of radiation. Isodose lines show radiation dose: green $100 \%$, red $97 \%$, white $95 \%$, orange $90 \%$.

fractions. Preclinical data, with clinical data to follow, suggest that dose escalation overcomes RCC radioresistance.

\section{Dose Escalation for SRS in Intracranial Metastatic RCC}

Autopsy series suggest that $10 \%$ of patients with RCC have brain metastases at death. Standard treatment for RCC brain metastases has been wholebrain radiotherapy (WBRT), but survival is poor. Data from MD Anderson indicate that the median survival for patients with a solitary metastasis is 4.4 months compared with 3.0 months in those with multiple metastases. Mortality was driven by neurologic death rather than systemic progression. ${ }^{25}$

The Radiation Therapy Oncology Group (RTOG) used recursive partitioning analysis (RPA) to create prognostic groups based on patient and treatment characteristics, including age, Karnofsky performance status, and extracranial disease control. ${ }^{26}$ Patients in RPA class 1 have the best overall prognosis. Data from Rades et $\mathrm{al}^{27}$ suggest that WBRT doses greater than 30 Gy given over 10 fractions improve 1 -year overall survival, even in patients in RTOG RPA class 2 and 3, with a trend toward improved intracranial control. In a Cleveland Clinic series, patients receiving more than 30 Gy of WBRT survived longer than those receiving 30 Gy or 

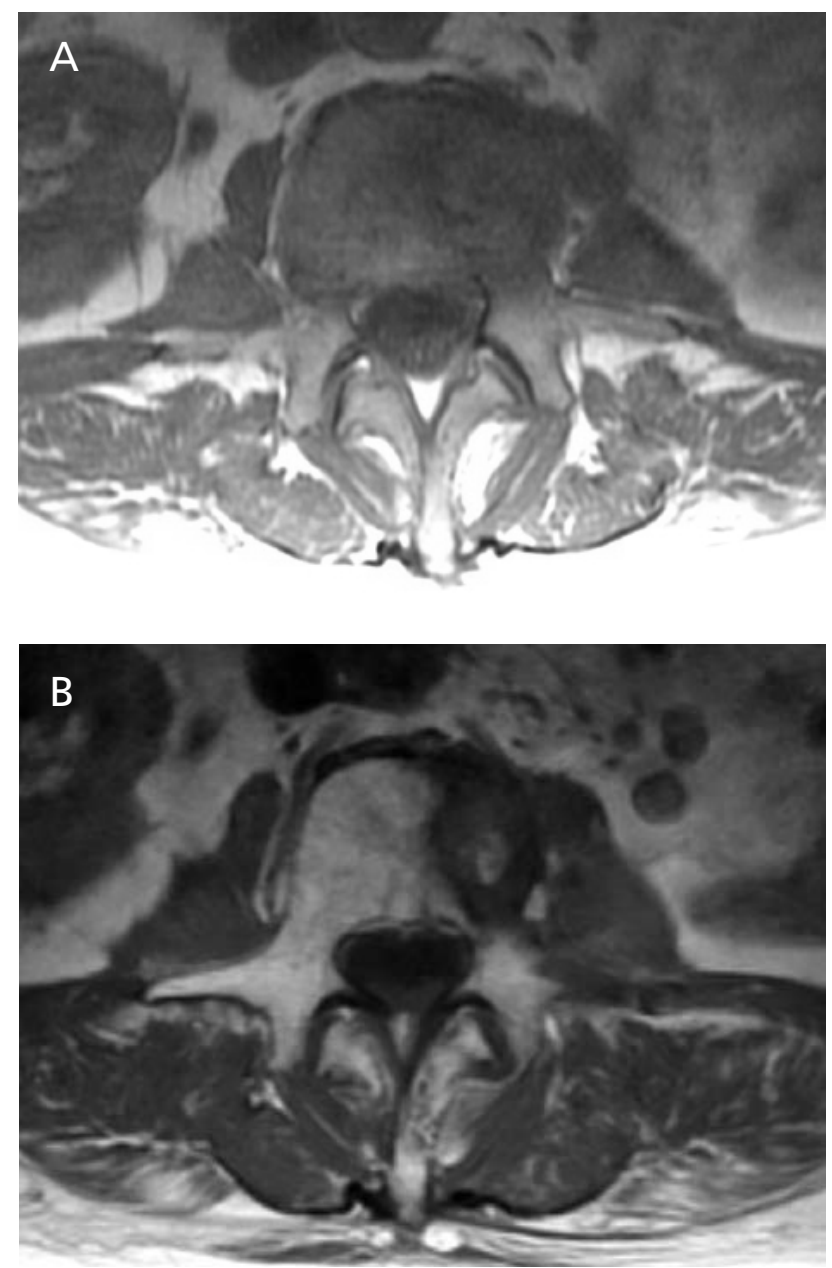

Figure 2 T1-weighted axial MRI in 74-year-old woman showing (A) L3 body and pedicle disease with paraspinal extension and (B) stable disease 8 months after treatment with 24 Gy in a single fraction.

less. ${ }^{28}$ Multiple prospective phase III trials using WBRT +/- SRS and SRS +/- WBRT suggest that dose escalation may improve local control (in multiple histologies) without necessarily improving overall survival. ${ }^{29-31}$

These data indicate that patients with RCC who are medically unfit for surgery, have multiple metastases, or have inoperable disease may benefit from higher-dose RT. Brown et $\mathrm{a}^{32}$ reviewed 83 brain metastases in 41 patients treated with SRS for radioresistant tumors (RCC, melanoma, and sarcoma); 16 of 41 had RCC and $44 \%$ had solitary metastasis. Patients in RPA class I had median survival of 23.5 months. Actuarial local control was $86 \%$ at 1 year. Standard WBRT was not sufficient for effective intracranial control. High-dose RT using highly targeted SRS up to 24 Gy offers local control as high as $90 \%$, with the possibility of omitting WBRT in select patients. ${ }^{32-34}$

\section{Extracranial SRS for Metastatic Spine Lesions}

Extracranial SRS, also known as SBRT or stereotactic ablative body radiotherapy, applies the same ablative potential to body lesions. Early data from the Karolinska Institute yielded excellent local control in patients with RCC metastases to lung, liver, and bone. In 162 lesions treated in 50 patients from 1997 to 2003, local control rates were greater than $90 \%$. Dose varied from 5 Gy x 5 fractions to 16 Gy $\mathrm{x} 2$ fractions, but all patients were treated with stereotactic immobilization, multiple conformal beams, and custom multileaf collimation. Interestingly, patients with 1 to 3 metastases survived longer than those with more than 3 metastases, suggesting that local therapy may confer a survival benefit in the oligometastatic setting, particularly in patients with reduced disease burden. ${ }^{11}$

The spine is the most common site of osseous metastatic disease in patients with cancer, and approximately $40 \%$ have spinal disease at death. ${ }^{35} \mathrm{~A}$ minority of spine lesions will become symptomatic, but pain and neurologic compromise can develop rapidly and be catastrophic. Conventional RT with various treatment schedules uses generous margins around the gross tumor volume, indiscriminately irradiating normal tissue, including the spinal cord. Immediate and durable palliation is often limited to radiosensitive tumors, such as breast and prostate carcinomas. ${ }^{14}$

SBRT requires several special techniques to deliver ablative RT safely and effectively, including (1) use of multiple conformal beams with intensitymodulation, (2) accuracy within millimeters, (3) image guidance with each treatment, and (4) custom immobilization. Multiple beams allow for shaping of highly conformal dose, particularly sparing the spinal cord, which is usually within millimeters of the target volume (Figure 1). ${ }^{36}$ Custom immobilization requires comfortable, reproducible patient positioning while securely immobilizing the shoulders, neck, abdomen, or pelvis, as needed ${ }^{37}$ Image guidance uses daily onboard imaging, ideally with pretreatment cone-beam CT. Further refinements include use of flatteningfilter-free technology to quickly deliver treatment, 
Spine Radiosurgery in Renal Cell Carcinoma

and a 6-degree-of-freedom treatment couch, which allows for correction of positioning errors in any plane.

Spinal cord identification is critical to allow for accurate estimation of cord dose. This is particularly important in the reirradiation setting when initial treatment fails to provide durable palliation..$^{38}$ The preferred approach at MSKCC is for all patients to undergo a presimulation CT-myelogram, unless contraindicated, using O-MAR (orthopedic metal artifact reduction), especially postoperative cases with implanted spinal hardware. O-MAR is a radiologic software algorithm (Philips Healthcare, Amsterdam, The Netherlands) that reduces artifact generated by metal and other high- $Z$ material. The authors found this method superior to image fusion with MRI, because MRI itself is prone to artifact from implanted hardware, and imperfect image fusion of MRI with the CT simulation scan may occur. Other institutions have found success using fusion of T2-weighted imaging, which may be easier to practice in the community setting. These technical advances allow delivery of high-dose RT while respecting cord tolerance. The authors use the Spratt Six Segmentation method to delineate the treatment volume, which treats gross tumor, abnormal marrow signal, and an adjacent normal bony segment to account for subclinical tumor. ${ }^{39}$

Between 2004 and 2010, MSKCC treated 105 RCC metastases (59 spine lesions) with single-dose SRS or hypofractionated SRS. The overall 3-year actuarial local progression-free survival rate was $44 \%$. In patients with disease treated in a single fraction and with a dose of 24 Gy or greater, the 3-year local progression-free survival rate was $88 \%$. In contrast, patients receiving hypofractionated treatment in 3 or 5 fractions had a 3-year local control rate of $17 \%$. Treatment delivered in a single fraction and with a dose of $24 \mathrm{~Gy}$ or greater significantly improved local control in multivariate analysis. ${ }^{40}$

Other series confirm excellent results, although many are mixed tumor series. Gerszten et al ${ }^{41,42}$ prospectively evaluated 500 lesions in 393 patients using radiosurgery for pain control; 344 lesions were previously radiated. All patients were treated with CyberKnife radiosurgery (Accuray Incorporated, Sunnyvale, CA) with a mean dose 20 Gy in one fraction. With a median follow-up of 21 months, the long-term pain improvement and radiographic control rates were $86 \%$ and $88 \%$, respectively. The 93 lesions in patients with RCC had slightly better pain improvement of 94\%, with $87 \%$ achieving radiographic control. No acute or subacute myelopathy was seen. Nguyen et al ${ }^{43}$ studied spine SRS for RCC in 55 lesions; $58 \%$ had prior radiation with median dose 30 Gy. A total of 8 lesions were treated with 24 Gy in 1 fraction, 34 with 27 Gy in 3 fractions, and 13 with 30 Gy in 5 fractions. Actuarial 1-year progression-free survival was $82.1 \%$; $64 \%$ of patients were pain-free at 9 months, a number that increased steadily after completing SRS. ${ }^{43}$

Hypofractionated and single-fraction approaches are both used in spine SRS. MSKCC published a report on 93 patients with 103 lesions treated with a median of $24 \mathrm{~Gy}$ in a single fraction; 21 lesions were RCC. The cord was limited to a 14-Gy maximum dose. Actuarial local control was 90\%. Patients treated with more than 23 Gy had improved local control compared with those treated with a lower dose. ${ }^{5}$ The Cleveland Clinic reported a similar series of singlefraction treatments in which 57 patients with 88 RCC lesions were treated with median dose of 15 Gy in 1 fraction, including approximately $20 \%$ receiving prior in-field RT. ${ }^{44}$ With a median follow-up of 5.4 months, the actuarial radiographic progression-free survival rate was $92.3 \%$ and $71.2 \%$ at 3 and 12 months, respectively, and the adjusted pain-free survival rate was $84.9 \%$ and $67.7 \%$ at 3 and 12 months, respectively.

An important point to highlight is the durability of SRS in serial MRI follow-up or clinical symptom assessment, particularly with single-fraction treatment (Table 1). The authors' institutional experience indicates an actuarial 3-year local control rate of greater than $90 \%$ in patients treated with highdose single-fraction treatment. ${ }^{5,40}$ Other series have consistently reported local control 1 to 3 years after treatment at more than $80 \% .42,43$ Given that patients with RCC are living longer and systemic therapy is improving, durable control is critical.

Prospective evaluation has largely been limited to single-institution experiences. ${ }^{45} \mathrm{~A}$ phase II/III study (RTOG-0631) of spine SRS has already established the feasibility of studying this technique in a multi-institutional cooperative group setting. ${ }^{46}$ The International Spine Radiosurgery Consortium has published consensus guidelines for spine SRS target volumes. ${ }^{39}$ These cooperative groups will further standardize technique, outcome, and toxicity reporting in future trials.

Early reports indicate the relative safety of the treatment, even with previous in-field RT. The most 
Taunk et al

Table 1 Selected Series for Spine Stereotactic Radiosurgery in RCC and Mixed Histologies

\begin{tabular}{|c|c|c|c|c|c|c|c|}
\hline Reference & Patients & Lesions & Histology & Dose (Gy) & $\begin{array}{l}\text { Treatment } \\
\text { Fractions }\end{array}$ & $\begin{array}{l}\text { Local } \\
\text { Control }\end{array}$ & $\begin{array}{l}\text { Follow-Up } \\
\text { (Actuarial) }\end{array}$ \\
\hline Balagamwala et al, ${ }^{44} 2012$ & 57 & 88 & $\mathrm{RCC}$ & 15 & 1 & $71.2 \%$ & $12 \mathrm{mo}$ \\
\hline Gerszten et al, ${ }^{42} 2005$ & 48 & 60 & $\mathrm{RCC}$ & 20 (mean) & 1 & $89.0 \%$ & $\begin{array}{l}37 \text { mo } \\
\text { (median) }\end{array}$ \\
\hline Gerszten et al, ${ }^{41} 2007$ & 393 & 500 & Mixed & 20 (mean) & 1 & $88.0 \%$ & $\begin{array}{l}21 \text { mo } \\
\text { (median) }\end{array}$ \\
\hline Sohn et al, ${ }^{59} 2014$ & 13 & 13 & $\mathrm{RCC}$ & $\begin{array}{l}38 \text { (marginal } \\
\text { dose) }\end{array}$ & $1-5$ & $85.7 \%$ & $12 \mathrm{mo}$ \\
\hline Thibault et al, ${ }^{60} 2014$ & 37 & 71 & $\mathrm{RCC}$ & 24 & 2 & $83.0 \%$ & $12 \mathrm{mo}$ \\
\hline Wang et al, ${ }^{45} 2012$ & 149 & 166 & Mixed & $27-30$ & 3 & $80.5 \%$ & $12 \mathrm{mo}$ \\
\hline Yamada et al, ${ }^{5} 2008$ & 93 & 103 & Mixed & 24 & 1 & $90.0 \%$ & $15 \mathrm{mo}$ \\
\hline Zelefsky et al, ${ }^{40} 2012$ & 45 & 45 & $\mathrm{RCC}$ & 24 & 1 & $88.0 \%$ & $36 \mathrm{mo}$ \\
\hline
\end{tabular}

Abbreviation: RCC, renal cell carcinoma.

feared toxicity is myelopathy due to exceeding cord tolerance or errors in treatment positioning. However, this is an uncommon complication, and many series report no myelopathy. The Cleveland Clinic series $^{44}$ reports a nearly $5 \%$ rate of grade 1 and 2 motor neuropathy, whereas the Nguyen series ${ }^{43}$ reports a $2 \%$ rate of grade 1 toxicity. A Stanford series ${ }^{47}$ using CyberKnife reported 3 of 62 patients with severe myelopathy. Interestingly, all complications in the Stanford series were for thoracic spine lesions, and 2 had prior exposure to targeted VEGF therapy. This finding highlights the imperfect understanding of cord tolerance, particularly with regard to prior treatment and systemic therapy. The Gerszten RCC series $^{42}$ and the authors' own MSKCC published data indicate no acute myelopathy. ${ }^{5,36,42-44}$

Vertebral compression fracture (VCF) is reported with higher incidence. Sahgal et $\mathrm{al}^{48}$ reported on 252 patients with 410 lesions treated with spine SRS. They report a cumulative incidence of VCF at $13.49 \%$ at 2 years. The greatest risk factors for VCF in multivariate analysis were high dose per fraction ( $\geq 24$ Gy and 20-23 Gy), baseline VCF, lytic disease, and preexisting spinal deformity. A total of $43 \%$ of lesions required salvage kyphoplasty, percutaneous screw stabilization, or surgery. ${ }^{48}$ Other series report similar rates of VCF, ranging from $11 \%$ to $20 \% .{ }^{49,50}$ The authors' own data indicate radiologic VCF in up to $39 \%$ of patients, independent of histology. Symptoms primarily required increased use of narcotic medication, but only 2 of 27 patients with fracture progression required surgery or kyphoplasty. These data indicate that lesions that are lytic, located between T10 and the sacrum, and have increased percentage of vertebral body involvement are more likely to experience VCF ${ }^{51}$ Cunha et $\mathrm{a}^{150}$ also reported that lytic lesions and greater than 20 Gy per fraction are associated with increased risk of fracture. Although most VCFs can be conservatively managed, further characterization is certainly warranted given heterogeneity in reporting, follow-up, and intervention. ${ }^{48}$

\section{Spine SRS and Targeted Therapy}

Targeted therapies are critical in the management of patients with metastatic RCC, particularly medications such as sunitinib, an oral anti-VEGF tyrosine kinase inhibitor, and temsirolimus, an intravenous mTOR inhibitor. The data are inconclusive and under active study, but antiangiogenic agents specifically may induce a window of vascular normalization that may have a synergistic antitumor effect with RT. However, the exact timing of drug administration (before RT, concurrently, or after RT) to maximize benefit has not yet been determined. ${ }^{52}$ Any synergistic effect using highdose treatment is likely caused by another mechanism, given the ablative nature of SRS compared with fractionated RT. Staehler et $\mathrm{al}^{53}$ reported on a large series of patients with RCC spine or brain metastases treated concurrently with SRS, plus sunitinib or sorafenib. There was only grade 1 abdominal pain in a patient with treatment to the spine (median, $20 \mathrm{~Gy}$ ) and local control was $90.4 \%$ at 24 months. Additional prospective series report on the safety on concurrent sunitinib and RT, although the number of patients with RCC 
in these studies is small. ${ }^{54,55}$ The authors' practice is to hold targeted therapy 3 to 5 days before SRS, and resume 3 to 5 days after treatment. Although some early series do report safety of concurrent targeted therapy and radiation, the authors cannot recommend routine concurrent therapy outside of a clinical trial without understanding potential toxicity.

\section{Emerging Issues and Conclusions}

Local control is the most meaningful outcome, but there are several ways to report this, including radiographic response and physician- or patient-reported pain response. Consensus reporting criteria are important to establish. Early series report on mixed tumor types and prior therapy, including prior RT, decompressive surgery, or mechanical stabilization. Reporting of increasingly homogenous cohorts is expected as adoption of spine SRS improves. Toxicity reporting is also important, but it is heterogeneous based on the location of disease, such as severe dysphagia for disease in the cervical spine compared with nausea for disease in the lumbar spine. Finally, consensus will have to emerge with respect to prescription dose, normal tissues constraints, and target volume definition. ${ }^{39,56}$

The authors' practice is to recommend SRS alone in patients with oligometastatic disease and mechanically stable spines. Operating in the NOMS (Neurologic, Oncologic, Mechanical instability, and Systemic disease) clinical framework, patients with spine lesions are assessed in a multidisciplinary clinic at MSKCC by a radiation oncologist, spine neurosurgeon, and neurointerventional radiologist. ${ }^{57}$ Careful patient selection is critical to identify those who may benefit the most from treatment, including patients with radioresistant diseases and those for whom prior radiation treatment failed (Figure 3). Indicated procedures are performed for stabilization using implanted hardware or kyphoplasty before radiation. Patients with RCC who present with highgrade spinal cord compression often require surgical decompression and stabilization to separate the tumor from the spinal cord and facilitate delivery of SRS while remaining within spinal cord tolerance. ${ }^{58}$ Patients can be treated with SRS and followed up with serial MRIs.

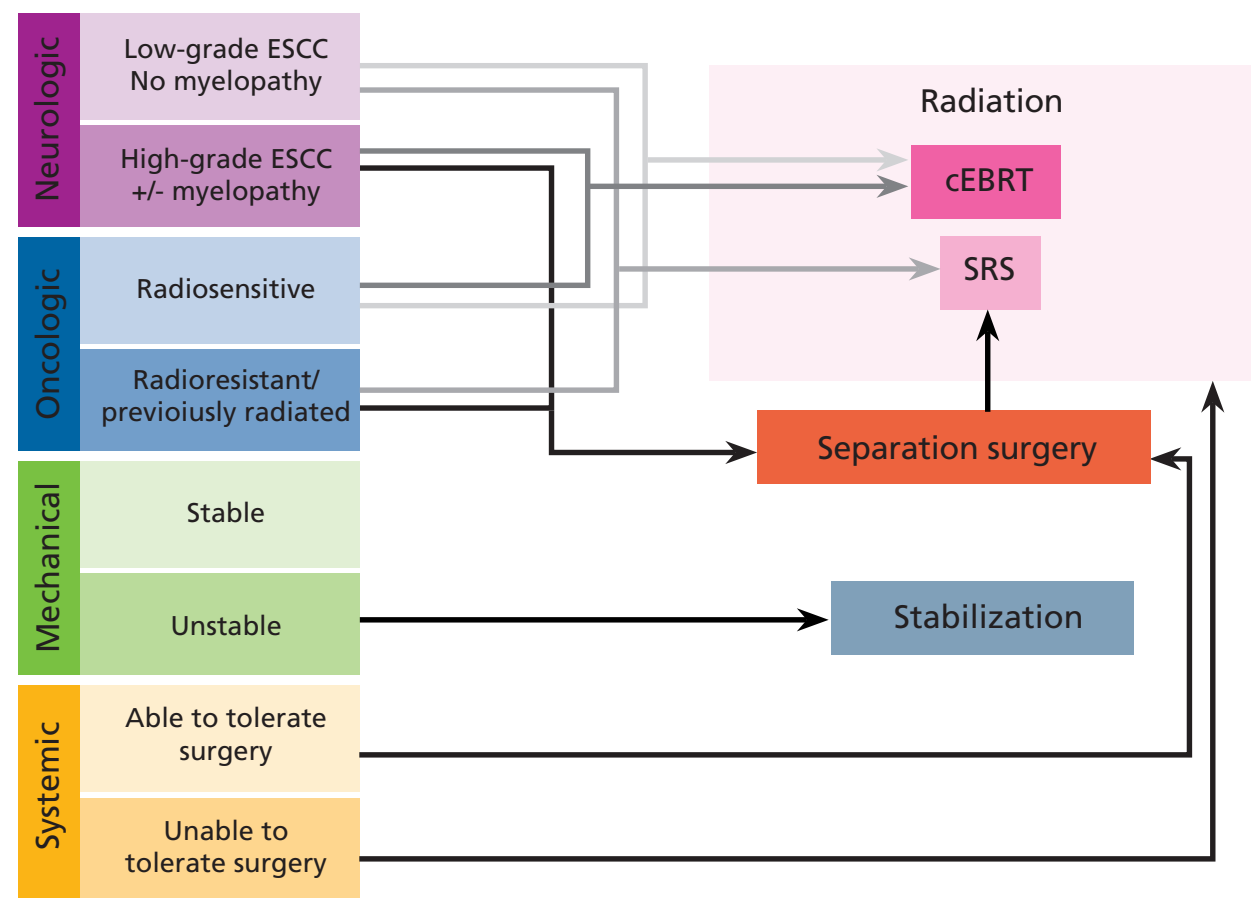

Figure 3 The NOMS clinical framework for SRS, surgery, and conventional radiation therapy in treating patients with spine metastasis. Abbreviations: ESCC, epidural spinal cord compression; CEBRT, conventional fractionated external-beam RT; NOMS, Neurologic, Oncologic, Mechanical, Systemic; SRS, stereotactic radiosurgery.

Adapted from Laufer I, Rubin DG, Lis E, et al. The NOMS framework: approach to the treatment of spinal metastatic tumors. Oncologist 2013;18:750; with permission. 
Taunk et al

Spine radiosurgery is an effective tool in managing patients with RCC. Although RT has little role in the treatment of primary disease, SRS does play an important role in the treatment of patients with spinal metastases, particularly those who received prior RT or instrumentation. It is known from multiple series that spine SRS for RCC has extremely high rates of durable local control and palliation. However, it demands high quality control, precision guidance, and careful patient selection to be safely and effectively implemented.

\section{References}

1. Motzer RJ, Bander NH, Nanus DM. Renal-cell carcinoma. N Engl J Med 1996;335:865-875.

2. Woodward E, Jagdev S, McParland L, et al. Skeletal complications and survival in renal cancer patients with bone metastases. Bone 2011;48:160-166.

3. McKay RR, Kroeger N, Xie W, et al. Impact of bone and liver metastases on patients with renal cell carcinoma treated with targeted therapy. Eur Urol 2014;65:577-584.

4. Motzer RJ, Mazumdar M, Bacik J, et al. Survival and prognostic stratification of 670 patients with advanced renal cell carcinoma. J Clin Oncol 1999;17:25302540.

5. Yamada Y, Bilsky MH, Lovelock DM, et al. High-dose, single-fraction imageguided intensity-modulated radiotherapy for metastatic spinal lesions. Int J Radiat Oncol Biol Phys 2008;71:484-490.

6. Flanigan RC, Mickisch G, Sylvester R, et al. Cytoreductive nephrectomy in patients with metastatic renal cancer: a combined analysis. J Urol 2004;171:1071-1076.

7. Juusela H, Malmio K, Alfthan O, Oravisto KJ. Preoperative irradiation in the treatment of renal adenocarcinoma. Scand J Urol Nephrol 1977;11:277-281.

8. van der Werf-Messing B. Carcinoma of the kidney. Cancer 1973;32:1056-1061.

9. Kjaer M, Frederiksen PL, Engelholm S. Postoperative radiotherapy in stage II and III renal adenocarcinoma. A randomized trial by the Copenhagen renal cancer study group. Int J Radiat Oncol Biol Phys 1987;13:665-672.

10. Tunio MA, Hashmi A, Rafi M. Need for a new trial to evaluate postoperative radiotherapy in renal cell carcinoma: a meta-analysis of randomized controlled trials. Ann Oncol 2010;21:1839-1845.

11. Wersäll PJ, Blomgren $H$, Lax I, et al. Extracranial stereotactic radiotherapy for primary and metastatic renal cell carcinoma. Radiother Oncol 2005;77:88-95.

12. Beitler JJ, Makara D, Silverman P, Lederman G. Definitive, high-dose-perfraction, conformal, stereotactic external radiation for renal cell carcinoma. Am J Clin Oncol 2004;27:646-648.

13. Siva S, Pham D, Gill S, et al. A systematic review of stereotactic radiotherapy ablation for primary renal cell carcinoma. BJU Int 2012;110:E737-743.

14. Hartsell WF, Scott CB, Bruner DW, et al. Randomized trial of short- versus long-course radiotherapy for palliation of painful bone metastases. J Natl Cancer Inst 2005;97:798-804.

15. Wang JZ, Guerrero M, Li XA. How low is the $\alpha / \beta$ ratio for prostate cancer? Int J Radiat Oncol Biol Phys 2003;55:194-203.

16. Ning S, Trisler K, Wessels BW, Knox SJ. Radiobiologic studies of radioimmunotherapy and external beam radiotherapy in vitro and in vivo in human renal cell carcinoma xenografts. Cancer 1997;80(12 Suppl):2519-2528.

17. Deschavanne PJ, Fertil B. A review of human cell radiosensitivity in vitro. Int J Radiat Oncol Biol Phys 1996;34:251-266.

18. Walsh L, Stanfield JL, Cho LC, et al. Efficacy of ablative high-dose-per-fraction radiation for implanted human renal cell cancer in a nude mouse model. Eur Urol 2006;50:795-800.

19. Haimovitz-Friedman A, Kan CC, Ehleiter D, et al. Ionizing radiation acts on cellular membranes to generate ceramide and initiate apoptosis. J Exp Med 1994;180:525-535.

20. Garcia-Barros M, Paris F, Cordon-Cardo C, et al. Tumor response to radiotherapy regulated by endothelial cell apoptosis. Science 2003;300:1155-1159.

21. DiBiase SJ, Valicenti RK, Schultz D, et al. Palliative irradiation for focally symptomatic metastatic renal cell carcinoma: support for dose escalation based on a biological model. J Urol 1997;158:746-749.

22. Brenner DJ. The linear-quadratic model is an appropriate methodology for determining isoeffective doses at large doses per fraction. Semin Radiat Oncol 2008;18:234-239.
23. Kirkpatrick JP, Meyer JJ, Marks LB. The linear-quadratic model is inappropriate to model high dose per fraction effects in radiosurgery. Semin Radiat Oncol 2008;18:240-243.

24. Park C, Papiez L, Zhang S, et al. Universal survival curve and single fraction equivalent dose: useful tools in understanding potency of ablative radiotherapy. Int J Radiat Oncol Biol Phys 2008;70:847-852.

25. Wrónski M, Maor MH, Davis BJ, et al. External radiation of brain metastase from renal carcinoma: a retrospective study of 119 patients from the M. D. Anderson Cancer Center. Int J Radiat Oncol Biol Phys 1997;37:753-759.

26. Gaspar L, Scott C, Rotman M, et al. Recursive partitioning analysis (RPA) of prognostic factors in three Radiation Therapy Oncology Group (RTOG) brain metastases trials. Int J Radiat Oncol Biol Phys 1997;37:745-751.

27. Rades D, Heisterkamp C, Schild SE. Do patients receiving whole-brain radiotherapy for brain metastases from renal cell carcinoma benefit from escalation of the radiation dose? Int J Radiat Oncol Biol Phys 2010;78:398-403.

28. Cannady SB, Cavanaugh KA, Lee SY, et al. Results of whole brain radiotherapy and recursive partitioning analysis in patients with brain metastases from renal cell carcinoma: a retrospective study. Int J Radiat Oncol Biol Phys 2004;58:253-258.

29. Kocher M, Soffietti R, Abacioglu U, et al. Adjuvant whole-brain radiotherapy versus observation after radiosurgery or surgical resection of one to three cerebral metastases: results of the EORTC 22952-26001 study. J Clin Oncol 2011;29:134-141.

30. Kondziolka D, Patel A, Lunsford LD, et al. Stereotactic radiosurgery plus whole brain radiotherapy versus radiotherapy alone for patients with multiple brain metastases. Int J Radiat Oncol Biol Phys 1999;45:427-434.

31. Andrews DW, Scott CB, Sperduto PW, et al. Whole brain radiation therapy with or without stereotactic radiosurgery boost for patients with one to three brain metastases: phase III results of the RTOG 9508 randomised trial. Lancet 2004;363:1665-1672.

32. Brown PD, Brown CA, Pollock BE, et al. Stereotactic radiosurgery for patients with "radioresistant" brain metastases. Neurosurgery 2002;51:656-665.

33. Goyal LK, Suh JH, Reddy CA, Barnett GH. The role of whole brain radiotherapy and stereotactic radiosurgery on brain metastases from renal cell carcinoma. Int J Radiat Oncol Biol Phys 2000;47:1007-1012.

34. Manon R, O'Neill A, Knisely J, et al. Phase II trial of radiosurgery for one to three newly diagnosed brain metastases from renal cell carcinoma, melanoma, and sarcoma: an Eastern Cooperative Oncology Group study (E 6397). J Clin Oncol 2005;23:8870-8876.

35. Wong DA, Fornasier VL, MacNab I. spinal metastases: the obvious, the occult, and the impostors. Spine 1990;15:1-4.

36. Yamada Y, Lovelock DM, Yenice KM, et al. Multifractionated image-guided and stereotactic intensity-modulated radiotherapy of paraspinal tumors: a preliminary report. Int J Radiat Oncol Biol Phys 2005;62:53-61.

37. Chang EL, Shiu AS, Mendel E, et al. Phase I/II study of stereotactic body radiotherapy for spinal metastasis and its pattern of failure. J Neurosurg Spine 2007;7:151-160.

38. Chow E, Danjoux C, Wong R, et al. Palliation of bone metastases: a survey of patterns of practice among Canadian radiation oncologists. Radiother Oncol 2000;56:305-314

39. Cox BW, Spratt DE, Lovelock M, et al. International Spine Radiosurgery Consortium consensus guidelines for target volume definition in spinal stereotactic radiosurgery. Int J Radiat Oncol Biol Phys 2012;83:e597-605.

40. Zelefsky MJ, Greco C, Motzer R, et al. Tumor control outcomes after hypofractionated and single-dose stereotactic image-guided intensitymodulated radiotherapy for extracranial metastases from renal cell carcinoma. Int J Radiat Oncol Biol Phys 2012;82:1744-1748.

41. Gerszten PC, Burton SA, Ozhasoglu C, Welch WC. Radiosurgery for spinal metastases: clinical experience in 500 cases from a single institution. Spine (Phila Pa 1976) 2007;32:193-199.

42. Gerszten PC, Burton SA, Ozhasoglu C, et al. Stereotactic radiosurgery for spinal metastases from renal cell carcinoma. J Neurosurg Spine 2005;3:288-295.

43. Nguyen $\mathrm{QN}$, Shiu AS, Rhines LD, et al. Management of spinal metastases from renal cell carcinoma using stereotactic body radiotherapy. Int J Radiat Oncol Biol Phys 2010;76:1185-1192.

44. Balagamwala EH, Angelov L, Koyfman SA, et al. Single-fraction stereotactic body radiotherapy for spinal metastases from renal cell carcinoma. J Neurosurg Spine 2012;17:556-564.

45. Wang XS, Rhines LD, Shiu AS, et al. A prospective analysis of the clinical effects of stereotactic body radiation therapy in cancer patients with spinal metastases without spinal cord compression. Lancet Oncol 2012;13:395-402.

46. Ryu S, Pugh SL, Gerszten PC, et al. RTOG 0631 phase $2 / 3$ study of image guided stereotactic radiosurgery for localized (1-3) spine metastases: phase 2 results. Pract Radiat Oncol 2014;4:76-81.

47. Gibbs IC, Kamnerdsupaphon $P$, Ryu MR, et al. Image-guided robotic radiosurgery for spinal metastases. Radiother Oncol 2007;82:185-190. 
Spine Radiosurgery in Renal Cell Carcinoma

48. Sahgal A, Whyne CM, Ma L, et al. Vertebral compression fracture after stereotactic body radiotherapy for spinal metastases. Lancet Oncol 2013;14:e310-320

49. Boehling NS, Grosshans DR, Allen PK, et al. Vertebral compression fracture risk after stereotactic body radiotherapy for spinal metastases. J Neurosurg Spine 2012;16:379-386

50. Cunha MV, Al-Omair A, Atenafu EG, et al. Vertebral compression fracture (VCF) after spine stereotactic body radiation therapy (SBRT): analysis of predictive factors. Int J Radiat Oncol Biol Phys 2012;84:e343-349.

51. Rose PS, Laufer I, Boland PJ, et al. Risk of fracture after single fraction imageguided intensity-modulated radiation therapy to spinal metastases. J Clin Oncol 2009; $27: 5075-5079$

52. Mazeron R, Anderson B, Supiot S, et al. Current state of knowledge regarding the use of antiangiogenic agents with radiation therapy. Cancer Treat Rev 2011;37:476-486.

53. Staehler M, Haseke N, Nuhn P, et al. Simultaneous anti-angiogenic therapy and single-fraction radiosurgery in clinically relevant metastases from renal cell carcinoma. BJU Int 2011;108:673-678.

\section{Instructions for Completion}

To participate in this journal CE activity: 1) review the learning objectives and author disclosures; 2 ) study the education content; 3 ) take the posttest with a $66 \%$ minimum passing score and complete the evaluation at http://education.ncon.org/ node/68461; and 4) view/print certificate. After reading the article, you should be able to answer the following multiple-

\section{Posttest Questions}

1. In patients with advanced RCC, RT is used for palliation of:

a. Painful bone metastases

b. Spinal cord compression

c. Brain metastases

d. All of the above

2 SRS alone can be recommended in carefully selected patients with:

a. Oligometastatic disease and mechanically stable spines

b. Oligometastatic disease and mechanically unstable spines
54. Tong CC, Ko EC, Sung MW, et al. Phase II trial of concurrent sunitinib and image-guided radiotherapy for oligometastases. PLoS ONE 2012;7:e36979.

55. Kao J, Packer S, Vu HL, et al. Phase 1 study of concurrent sunitinib and imageguided radiotherapy followed by maintenance sunitinib for patients with oligometastases. Cancer 2009;115:3571-3580.

56. Sahgal A, Larson DA, Chang EL. Stereotactic body radiosurgery for spinal metastases: a critical review. Int J Radiat Oncol Biol Phys 2008;71:652-665.

57. Laufer I, Rubin DG, Lis E, et al. The NOMS framework: approach to the treatment of spinal metastatic tumors. Oncologist 2013;18:744-751.

58. Laufer I, Iorgulescu JB, Chapman $T$, et al. Local disease control for spinal metastases following "separation surgery" and adjuvant hypofractionated or high-dose single-fraction stereotactic radiosurgery: outcome analysis in 186 patients. J Neurosurg Spine 2013;18:207-214.

59. Sohn S, Chung CK, Sohn MJ, et al. Stereotactic radiosurgery compared with external radiation therapy as a primary treatment in spine metastasis from renal cell carcinoma: a multicenter, matched-pair study. J Neurooncol 2014;119:121128.

60. Thibault I, Al-Omair A, Masucci GL, et al. Spine stereotactic body radiotherapy for renal cell cancer spinal metastases: analysis of outcomes and risk of vertebral compression fracture. J Neurosurg Spine 2014;21:711-718.

choice questions. Credit cannot be obtained for tests completed on paper. You must be a registered user on NCCN.org. If you are not registered on NCCN.org, click on "New Member? Sign up here" link on the left hand side of the Web site to register. Only one answer is correct for each question. Once you successfully answer all posttest questions you will be able to view and/or print your certificate. Software requirements: Internet

c. High-grade spinal cord compression

3. Highly targeted SRS using a high dose of up to ___ Gy has been shown to offer high local control (as high as $90 \%$ ), with the possibility of omitting WBRT in select patients.
a. 24
b. 32
c. 45

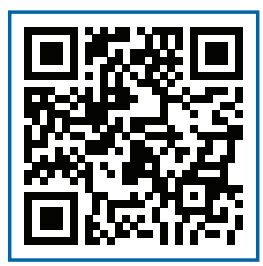

